\title{
Can Title Images Predict the Emotions and the Performance of Crowdfunding Projects?
}

\author{
Jian-Ren Hou \\ University of Texas at Arlington \\ jienren.hou@uta.edu
}

\author{
Jie Jennifer Zhang \\ University of Texas at Arlington \\ jiezhang@uta.edu
}

\author{
Kunpeng Zhang \\ University of Maryland \\ kzhang@rhsmith.umd.edu
}

\begin{abstract}
Crowdfunding is a novel way to raise funds from individuals. However, taking Kickstarter for example, more than $60 \%$ of projects failed to reach the funding targets. Hence it is imperative to study how to improve the successfulness of the projects. From a design perspective, we intend to investigate that can the characteristics of title images of the projects on the search page of the crowdfunding website predict the performance of crowdfunding projects. We use objective standards to measure the aesthetic features of the title images. And we introduce emotions as important antecedents for the performance of a project. We used deep learning to extract the emotion metrics from the title images. Analysis results provide significant evidence that aesthetic attributes of images can predict emotion in images, and emotions, such as sadness and contentment, can predict the performance of crowdfunding projects. Our results provide both theoretical and practical values.
\end{abstract}

\section{Introduction}

Crowdfunding is a kind of digital platform that raises funds by aggregating the contributions of many individuals to achieve a preset goal (Mollick, 2014; Ordanini et al., 2011). Crowdfunding has become a popular way to raise funds. As a leading crowdfunding platform that "helps bring creative projects to life", Kickstarter.com has raised over $\$ 3.4$ billion for 137,171 projects (Kickstarter Stats, Dec. 2017). Nonetheless, more than $60 \%$ of Kickstarter projects failed to reach their preset goals (Kickstarter Stats, Dec. 2017). Thus, it calls for attention to improve the successfulness of crowdfunding projects.

Echoing the needs, recent studies have focused on the influencing factors of the performance of crowdfunding projects such as design settings, e.g. duration, award amount, goal (Burtch et al., 2013; Mollick, 2014). This research how to improve the performance of crowdfunding projects from the perspective of title images. Title images of crowdfunding projects are the most attractive part when backers are browsing possible projects to pledge in search page. Researches provide evidence that images could possibly affect viewers' attitude and emotional reactions (Kelly et al., 2002). Findings of this research will suggest a fundraiser how to design the title image when launching a crowdfunding project.

We address three important and unresolved questions for funders in crowdfunding platform. First, can title images affect the performance of crowdfunding projects in terms of fund amount and participant number? Second, what attributes, such as composition, color, content, main body and background relationship, of the title images influence the performance of the crowdfunding projects, and how do they impact? Third, is emotion a critical factor between title images and the fundraising outcome?

Previous research suggests that picture could lead to higher quality beliefs for certain consumers, and inability of touching or holding true products makes this kind of visual cues more important (Peck \& Childers, 2003). Presenting the same object in different ways in photographs also affect viewers' emotional feeling (Baberini et al., 2015). Combination of users' positive or negative emotions could further inspire or motivate viewers to donate (Liang et al., 2016). Thus, emotions could be critical in successful charity crowdfunding campaigns. However, there still lack enough evidence or complete studies on how title images and their impacts on emotion influence crowdfunding performance. There are also some ecommerce studies (Bland et al. 2007, Di et al. 2014, and Goswami et al. 2011) have provided evidence that pictures do play an important role in terms of buyers' intention, trust, risk reduction, conversion rate, and click-through rate in the e-commerce scenario. However, the results from e-commerce scenario may not be applicable to crowdfunding. Moreover, we employ more tangible variables such as funds raised or number of backers. 
To address these questions, this study selects a category similar to charity, i.e., the public benefit category in crowdfunding, as the domain of research in the largest crowdfunding website --- Kickstarter.com. We choose this category due to the following reasons: 1) Americans donate an estimated of $\$ 390.05$ billion to charity in 2016, among which $\$ 281.86$ billion is donated by individuals (USA Giving, 2017). Given its main function of collecting funds from individuals, crowdfunding can be an effective way to raise funds for charity purposes or public benefits. 2) Literature on general charity fundraising has suggested related evidence to our hypotheses. For example, Baberini et al. (2015), Liang et al. (2016), and Small \& Verrochi (2009) showed that images expressing sadness to viewers could make viewers more likely to donate in charity fundraising projects.

We web scrape the title images on the listing page and project attribute data of all of the 842 projects in the public benefit category from the website of Kickstarter.com, including preset goals, participants numbers, total amount raised, and descriptive texts. To extract the emotion types (e.g. sadness, contentment, amusement) from those title images, we use deep learning technique to classify emotions in title images. To evaluate image property, we also employ Python image analysis package and image library to measure objective image attributes, such as composition, color, main body-ground relationship from images. To reflect the uniqueness of crowdfunding platform in our research model, we consider such variables as number of backers, amount raised, and pre-set goals.

This study contributes for related area in following ways. First, we are among the first studies that show emotions are important variables in connecting image attributes and crowdfunding outcomes. This research implements such objective measurements of image attributes and the resulted emotions which have been ignored in prior crowdfunding or charity/donation studies. Our results show that in the public benefit category of crowdfunding contests, title images attributes (e.g. composition, color, main body-ground relationship, content) influence emotions, which subsequently affect the performance of the projects. Second, we conduct complete and thorough analyses of the relationships between image attributes and crowdfunding outcomes. We use comprehensive tools, text and image analyses, to examine the emotional impact of creatives in crowdfunding projects, which display one new way in examining emotion related works. Third, we provide actionable and practical guides to crowdfunding funders and platforms on how to improve the performance through a more appropriate title image design.
The paper below is organized as follows: Section 2 summarizes and compares with the literature and proposes hypotheses; Section 3 introduces the data collection, variable definition and data statistics; Section 4 describes the model and presents the analysis results; Section 5 discusses the results to derive implications; and finally, Section 6 concludes the paper and discusses limitation and future research.

\section{Literature Review}

\subsection{Crowdfunding}

Traditionally, if someone needs certain amount of financial support to fulfill his/her proposal and related goal, he/she would probably need help from individual or organization. However, the growth of internet provides a new category of platform that allows individual or organization to seek financial support from considerable quantities of individuals. Although financial supports that individuals could provide are much less, thanks to the large number of individuals, crowdfunding platform still can provide efficient financial support for individual or organization who seeks help (Mollick, 2014; Ordanini et al., 2011).

Crowdfunding not only provides financial support for funders but also provides exposure chances for funder to draw more attention and awareness to crowdfunding projects (Burtch et al., 2013). In creativity industry such as film and music, crowdfunding provides not only financial support but also serves as a channel for makers to interact with their audience or receivers (Hobbs et al., 2016). Crowdfunding backers get characteristics of both traditional charitable donators and investors, moreover, some backers are motivated to pledge because their personal interests and preferences, which manifest the co-creation nature of crowdfunding (Ryu \& Kim, 2016). Although crowdfunding serves as a novel and innovative way for funders to seek financial support or possible additional value, fraud and deception are potential threats for funders or backers to pay attention to (Thürridl \& Kamleitner, 2016).

\subsection{Image}

Crowdfunding and e-commerce have many things in common. As an interface, websites would play the medium between users and platform, and users could not see the real product or outcome in person before paying their money. Some crowdfunding projects even be considered as pre-ordering. Thus, we believe that ecommerce researches could be considered as a good foothold for researchers to develop crowdfunding 
research. In the early stage of e-commerce research, study examines determinants which affect success of ecommerce mention that image should be considered in future research to further reveal more information regarding success (Gilkeson \& Reynolds, 2003). Further research investigates the effect of presence of real picture or stock picture, and the results indicate that presence of picture would positively influence the outcome of e-commerce (Bland et al., 2007). Furthermore, researchers not only consider the presence of picture but also include the quality features, such as brightness and contrast, and regional features, such as background and foreground setting (Goswami et al., 2011). These attributes are considered and are verified by researchers as important components which are related to click of e-commerce platform users (Goswami et al., 2011). Moreover, display of picture, picture count, and image quality could positively increase buyer's attention, trust and conversion rate (Di et al., 2014).

Images were commonly considered as emotion arousal in the literature (Carroll, 2003). Existing literature mention that emotional response is crucial while people react to artworks (Carroll, 2003). Literature believe that there is intimate relationship between art and emotions (Silvia, 2005). Emotions could be involved in aesthetic appreciation (Barry, 2006). On the other hand, positive and negative emotions are considered as important trigger of success fundraising (Baberini et al., 2015; Small \& Verrochi, 2009), but what attributes are actual antecedences of emotions are still questionable. This research doesn't connect image attributes to dependent variables directly. Instead of focusing only image or only emotion, we combine findings of past research to employ image attributes as antecedences of emotions and to enhance the image attributes by aesthetic features proposed by researchers (Wang et al., 2013; Zhang et al., 2017), and we propose hypothesis 1:

H1: Image attributes of title image can predict emotion in title images of crowdfunding projects.

Previous research indicates that when viewers see the photographs with sadness would increase viewers' donation amount, and emotion plays an important role in such mechanism (Small \& Verrochi, 2009). Further, different way to present the same object would lead to different emotion (Baberini et al., 2015). Negative emotion such as sadness could make people more willing to donate, but positive emotion could also inspire people to donate (Liang et al., 2016). Moreover, combination of both positive emotion and negative emotion could be effective to make people donate (Liang et al., 2016). Innovation is one key component of crowdfunding (Mollick \& Robb, 2016). For products which consumers didn't experience before, emotion plays an important role while consumer try to evaluate the focal product (Wood \& Moreau, 2006). Emotion is also significant in employee's implementation of innovations (Choi et al., 2011). Thus, we argue that emotions could be triggered by different attributes of title images and then predict crowdfunding fundraising outcome in terms of making backers more willing to provide financial support and adoption of innovation on crowdfunding platform.

On the other hand, text mining is a research field that multiple researches have focus on and many different algorithms are applied to mining emotion in such domain (Yadollahi et al., 2017). Some tools are considered reliable to detect emotion for text and been adapted by researchers, for example, LIWC matching target words in text content and its own dictionary of target words to measure emotion in certain text content (Pennebaker et al., 2015; Yin et al., 2014). Since not only visual cues such as images but also textual description affects the successfulness of crowdfunding, funders could include topical features in textual description and improve the possibility of successfulness (Yuan et al., 2016). Thus, we believe that emotion in text description is effective as well. Thus, we propose hypothesis $2 \mathrm{a}$ and $2 \mathrm{~b}$ of both text and image emotion's effect on crowdfunding performance.

H2a: Emotion in title images of crowdfunding projects can predict the performance of crowdfunding projects.

H2b: Emotion in textual description of crowdfunding projects can predict the performance of crowdfunding projects.

Thus, we propose the following model:

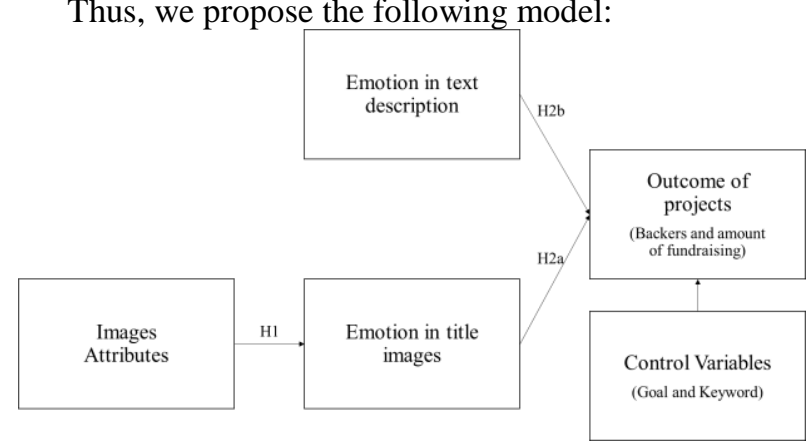

Figure 1. Research Model

Several existing works have empirically studied the possible relationship among text, image, emotion, or fundraising. We summarize some principal empirical 
researches to show the contribution of this study in Table 1.

Table 1. Summary of empirical principal literature review

\begin{tabular}{|l|c|c|c|c|c|}
\hline \multicolumn{1}{|c|}{ Article } & Image & Text & Emotion & $\begin{array}{c}\text { Outcome } \\
\text { (Fundraising, } \\
\text { e-commerce } \\
\text { performance) }\end{array}$ & $\begin{array}{c}\text { Crowd } \\
\text { funding }\end{array}$ \\
\hline $\begin{array}{l}\text { Machajdik } \\
\text { and } \\
\text { Hanbury } \\
2010\end{array}$ & $\mathrm{X}$ & $\mathrm{X}$ & & \\
\hline $\begin{array}{l}\text { Liang et al. } \\
2016\end{array}$ & & & $\mathrm{X}$ & $\mathrm{X}$ & \\
\hline $\begin{array}{l}\text { Small and } \\
\text { Verrochi } \\
2009\end{array}$ & $\mathrm{X}$ & $\mathrm{X}$ & $\mathrm{X}$ & \\
\hline $\begin{array}{l}\text { Yuan et al. } \\
2016\end{array}$ & $\mathrm{X}$ & $\mathrm{X}$ & $\mathrm{X}$ & $\mathrm{X}$ & $\mathrm{X}$ \\
\hline $\begin{array}{l}\text { Bland et al. } \\
2007\end{array}$ & $\mathrm{X}$ & $\mathrm{X}$ & $\mathrm{X}$ & $\mathrm{X}$ & $\mathrm{X}$ \\
\hline This Study & $\mathrm{X}$ & & & \\
\hline
\end{tabular}

\section{Data Collection}

We employ Python and web scraping packages as tools to collect data from Kickstarter.com. First, we use web scraping package to parse and extract necessary resource indexes for title images of each public benefit crowdfunding projects, and we download title images of crowdfunding projects from Kickstarter based on these indexes. Second, we employ text parsing technique and beautifulSoup-4 package to retrieve metrics for each project such as the number of backers, percentage of fundraising goal, and raised amount. We collect 842 images from our domain of research, public benefit. After removing 2 projects which were still under fundraising stage and not yet finished, there were 840 projects remained.

\subsection{Metrics Measurement}

3.1.1. Image metrics. This study follows the instrument of (Wang et al. 2013) and (Zhang et al. 2017). Above literatures provide a guideline for researchers and practitioners to judge the aesthetic characteristics of images objectively. It is worth mentioning that these attributes are just an objective way or standard to rate or judge images. However, meeting these standards doesn't mean that image is a good image, and vice versa. Even though, since aesthetic characteristics are mostly subjective, these objective standards still could provide values to researchers and practitioners. We list and propose conceptual definitions and operational definitions of these attributes we employ in this research in following section by the guideline provided by (Wang et al. 2013) and (Zhang et al. 2017).

We list all attributes we employ in this research in following table.

Table 2. Image Attributes and definitions

\begin{tabular}{|c|c|c|}
\hline Component & Attributes & Operational Definitions \\
\hline \multirow{4}{*}{ Composition } & $\begin{array}{l}\text { Diagonal } \\
\text { Dominance }\end{array}$ & $\begin{array}{l}\text { Distance between main } \\
\text { body and two diagonal } \\
\text { lines (lower is better) }\end{array}$ \\
\hline & Symmetry & $\begin{array}{l}\text { Is main body distributed } \\
\text { evenly on left and right } \\
\text { (lower is better) }\end{array}$ \\
\hline & Color Balance & $\begin{array}{l}\text { Is color distributed evenly } \\
\text { on left and right }\end{array}$ \\
\hline & Rule of Thirds & $\begin{array}{l}\text { Distance between main } \\
\text { body and two equally } \\
\text { spaced vertical lines } \\
\text { (lower is better) }\end{array}$ \\
\hline \multirow{4}{*}{ Color } & Warm Hue & $\begin{array}{l}\text { Pixels that color hue is } \\
\text { smaller than } 30 \text { and } \\
\text { bigger than } 110 \text { under } \\
\text { HSV color space }\end{array}$ \\
\hline & Saturation & Saturation \\
\hline & Brightness & Brightness \\
\hline & $\begin{array}{ll}\text { Contrast } & \text { of } \\
\text { Brightness } & \end{array}$ & Contrast of Brightness \\
\hline \multirow{3}{*}{$\begin{array}{l}\text { Main body- } \\
\text { ground } \\
\text { relationship }\end{array}$} & Size Difference & $\begin{array}{l}\text { Ratio of main body in } \\
\text { whole image }\end{array}$ \\
\hline & $\begin{array}{l}\text { Color } \\
\text { Difference }\end{array}$ & $\begin{array}{l}\text { Color difference between } \\
\text { main body and } \\
\text { background }\end{array}$ \\
\hline & $\begin{array}{l}\text { Texture } \\
\text { Difference }\end{array}$ & $\begin{array}{ll}\text { Texture } & \text { difference } \\
\text { between main body and } \\
\text { background }\end{array}$ \\
\hline
\end{tabular}

This study introduces the presence of people or even living creature in titles images as image attributes. On the other hand, evidences show that textual contents are effective to crowdfunding successfulness (Yuan et al. 2016). Hence, we also introduce the presence of text in title images as an image attribute. These attributes are recorded by two master's degree holder.

Table 3. Content of Images

\begin{tabular}{|l|l|}
\hline Attributes & Operational Definitions \\
\hline Text & $\begin{array}{l}\text { Are there any textual contents in the } \\
\text { images? }\end{array}$ \\
\hline Human & $\begin{array}{l}\text { Are there any human beings in the } \\
\text { images? }\end{array}$ \\
\hline Animal & $\begin{array}{l}\text { Are there any living creature other than } \\
\text { human in the images? }\end{array}$ \\
\hline
\end{tabular}




\subsubsection{Emotion metrics.}

\section{Emotion for Title Images:}

We employ the emotion set which contains: Amusement, Anger, Awe, Contentment, Disgust, Excitement, Fear, and Sadness from literature (Machajdik and Hanbury 2010). Different from Machajdik \& Hanbury (2010) who use low-level feature to classify emotion for picture, this study employs medium-level features to classify possible emotions for each title image. The medium-level features include adjective noun pairs (ANPs) and Google Vision API label are used. On the other hand, existing dataset of images are used to compare the performance of classification of medium-level features inputs. Based on these inputs and image dataset, deeplearning neural network classifier are trained. Results indicate that medium-level features do outperform lowlevel feature such as color, composition, and other features. We apply this trained neural network classifier on our crowdfunding title images dataset. The output of neural network classifier would provide degree of 8 emotions for each title images which ranges from 0 to 100 , and the degree increase when emotion increase. It's worth mention that our emotion measurements are not derived from any image attribute that we used as variables in our research. The emotion measurements are derived from solely deep learning technique. Thus, the measurements of image attributes and emotion is independent, which makes this study more valuable.

\section{Emotions for Text Description:}

We employ LIWC software (Pennebaker et al., 2015) to analyze the emotion in each crowdfunding projects' text description. The LIWC software analyze the degree of anxiety, anger, and sadness in text content. LIWC software has been widely adopted by researchers due to its reliability and validity to measure psychological component, such as emotion, from text content (Yin et al., 2014). Based on build-in dictionary, LIWC software could classify text content by matching words in its dictionary and words in text content. As each time match happens, LIWC would increase corresponding scale of matched category. After analyzing the percentage of each emotion word and term in all text content, LIWC would calculate the emotion score and the final score could be used to compare different emotional disclosure among different text content (Yin et al. 2014). Since research suggest that sadness would make people more willing to donate (Small and Verrochi 2009), we would like to investigate the effect of emotion, including sadness, in text description on crowdfunding performance in this study.

3.1.3. Crowdfunding metrics. Among measurements of successful crowdfunding, we select number of backers and amount of fundraising as dependent variables. The reason is that these measurements can provide easy and direct interpretations for readers.

Number of Backers: Backer is who contributes financial support to crowdfunding project. The unit is person for each finished crowdfunding project.

Amount: Total amount of money raised by each finished crowdfunding project. The unit is US dollar.

\section{Control Variables}

Preset Goal: Preset goal for each finished crowdfunding projects. Although preset goal is not the question of interest here, we also include it as a control variable since its possible effect on performance of crowdfunding projects.

Interest for Keywords: We use feature extraction package in Python programming language to extract top 10 popular nouns in text description for all crowdfunding projects in our dataset. Then we use Google Trends to get interest degree for each keyword (noun). By this procedure, we could know how popular for each keyword, and we could assign degree of interest for each crowdfunding project to control the possible effect that projects with popular (more interest) topic could have better performance than other projects.

\section{Results}

Since we choose two variables, number of backers and amount of fundraising, as our dependent variables, we would need to perform two separated path analyses to analyze the data. Following sections would show the results for models of each dependent variable.

\subsection{Model 1: Number of backers and Model 2: Amount of fundraising}

Since the path analysis would analyze the whole model simultaneously, we would need to report two models respectively. Following tables are results for model 1 and 2, which we use number of backers and amount raised as measurement of performance. Separately, Table 4 and Table 6 are results of model 1, and Table 5 and Table 7 are results of model 2 . 
Table 4. Effect of image attribute on image emotion (model 1)

\begin{tabular}{|c|c|c|c|c|c|c|c|}
\hline $\mathrm{IV} \quad \mathrm{DV}$ & $\begin{array}{c}\text { Amusement } \\
\mathrm{R}^{2}=6 \%\end{array}$ & $\begin{array}{c}\text { Awe } \\
\mathrm{R}^{2}=10.6 \%\end{array}$ & $\begin{array}{c}\text { Contentment } \\
\mathrm{R}^{2}=12.2 \%\end{array}$ & $\begin{array}{c}\text { Disgust } \\
\mathrm{R}^{2}=7.7 \%\end{array}$ & $\begin{array}{l}\text { Excitement } \\
\mathrm{R}^{2}=15.8 \%\end{array}$ & $\begin{array}{c}\text { Fear } \\
\mathrm{R}^{2}=6.7 \%\end{array}$ & $\begin{array}{c}\text { Sadness } \\
\mathrm{R}^{2}=6.3 \%\end{array}$ \\
\hline $\begin{array}{c}\text { Diagonal } \\
\text { Dominance }\end{array}$ & -0.004 & 0.171 & $\underline{-0.204 * * *}$ & 0.01 & 0.026 & 0.042 & 0.018 \\
\hline Symmetry & 0.000 & 0.000 & 0.001 & 0.001 & -0.001 & $\underline{-0.003 *}$ & -0.001 \\
\hline Color Balance & -0.009 & $0.088 * * *$ & 0.012 & $-0.076 * * *$ & 0.01 & 0.018 & -0.013 \\
\hline Rule of Thirds & 0.103 & $\underline{-0.194 * *}$ & 0.114* & 0.07 & -0.07 & -0.09 & 0.028 \\
\hline Warm Hue & 0.009 & -0.022 & -0.024 & -0.029 & $0.087 * * *$ & $\underline{-0.038 * *}$ & $\underline{-0.046 * * *}$ \\
\hline Saturation & 23.362** & 18.246* & $19.957 * * *$ & 16.991 & 37.273*** & $-34.433 * * *$ & $-37.842 * * *$ \\
\hline (Saturation) $^{2}$ & $-24.391 *$ & $-24.038^{*}$ & $-25.452 * * *$ & -13.702 & $-41.118 * * *$ & $35.508 * * *$ & $34.294 * * *$ \\
\hline Brightness & 0.001 & -0.02 & $0.018 * *$ & $0.034 * *$ & 0.006 & $\underline{-0.042 * * *}$ & $-0.022 * *$ \\
\hline $\begin{array}{l}\text { Contrast of } \\
\text { Brightness }\end{array}$ & -0.007 & $\underline{0.119 * *}$ & -0.016 & $\underline{-0.1^{*}}$ & 0.046 & 0.027 & $\underline{-0.076 * *}$ \\
\hline Size Difference & -0.063 & -0.413 & $\underline{0.408 * *}$ & 0.207 & $\underline{-0.478^{*}}$ & -0.152 & $\underline{0.4 * *}$ \\
\hline $\begin{array}{c}(\text { Size } \\
\text { Difference })^{2}\end{array}$ & 0.000 & 0.004 & $\underline{-0.006 *}$ & -0.003 & $\underline{0.008 *}$ & 0.000 & $\underline{-0.006 *}$ \\
\hline $\begin{array}{c}\text { Color } \\
\text { Difference }\end{array}$ & $-0.014 *$ & $-0.019 * *$ & -0.002 & 0.001 & -0.006 & 0.000 & 0.002 \\
\hline $\begin{array}{c}\text { Texture } \\
\text { Difference }\end{array}$ & 2.110 & -12.345 & 6.909 & -6.626 & 9.098 & 8.872 & 2.418 \\
\hline Text & $-4.201 * * *$ & $-6.605 * * *$ & $-2.732 * * *$ & -1.981 & -0.734 & 0.547 & -1.073 \\
\hline Human & $-2.167 *$ & $\underline{-5.599 * * *}$ & 0.435 & $-7.947 * * *$ & 12.154*** & $-2.009 * *$ & -0.902 \\
\hline Animal & $-7.379 * * *$ & -4.341 & $14.51 * * *$ & -3.774 & $\underline{-5.789 * *}$ & 0.51 & 1.910 \\
\hline
\end{tabular}

Table 5. Effect of image attribute on image emotion (model 2)

\begin{tabular}{|c|c|c|c|c|c|c|c|}
\hline $\mathrm{IV}$ DV & $\begin{array}{c}\text { Amusement } \\
\mathrm{R}^{2}=6 \%\end{array}$ & $\begin{array}{c}\text { Awe } \\
\mathrm{R}^{2}=10.6 \%\end{array}$ & $\begin{array}{c}\text { Contentment } \\
\mathrm{R}^{2}=12.2 \%\end{array}$ & $\begin{array}{c}\text { Disgust } \\
\mathrm{R}^{2}=7.7 \%\end{array}$ & $\begin{array}{l}\text { Excitement } \\
\mathrm{R}^{2}=15.8 \%\end{array}$ & $\begin{array}{c}\text { Fear } \\
\mathrm{R}^{2}=6.7 \%\end{array}$ & $\begin{array}{c}\text { Sadness } \\
\mathrm{R}^{2}=6.3 \%\end{array}$ \\
\hline $\begin{array}{c}\text { Diagonal } \\
\text { Dominance }\end{array}$ & -0.003 & 0.171 & $\underline{-0.204 * * *}$ & 0.01 & 0.026 & 0.042 & 0.018 \\
\hline Symmetry & 0.000 & 0.000 & 0.001 & 0.001 & -0.001 & $\underline{-0.003 *}$ & -0.001 \\
\hline Color Balance & -0.009 & $0.088 * * *$ & 0.012 & $-0.076 * * *$ & 0.01 & 0.018 & -0.012 \\
\hline Rule of Thirds & 0.103 & $-0.194 * *$ & 0.114* & 0.07 & -0.07 & -0.09 & 0.028 \\
\hline Warm Hue & 0.009 & -0.022 & -0.024 & -0.029 & $0.087 * * *$ & $\underline{-0.038 * *}$ & $-0.046 * * *$ \\
\hline Saturation & 23.786** & 18.093* & $19.952 * * *$ & 16.664 & $37.309 * * *$ & $-34.444 * * *$ & $-37.767 * * *$ \\
\hline$(\text { Saturation })^{2}$ & $\overline{-24.973 *}$ & $\overline{-23.776^{*}}$ & $-25.441 * * *$ & -13.274 & $-41.175 * * *$ & $35.522 * * *$ & $\overline{34.194 * * *}$ \\
\hline Brightness & 0.001 & -0.02 & $0.018 * *$ & $\underline{0.034 * * *}$ & 0.006 & $-0.042 * * *$ & $-0.022 * *$ \\
\hline $\begin{array}{l}\text { Contrast of } \\
\text { Brightness }\end{array}$ & -0.007 & $\underline{0.119 * *}$ & -0.016 & $\underline{-0.1 *}$ & 0.046 & 0.027 & $\underline{-0.076 * *}$ \\
\hline Size Difference & -0.064 & -0.413 & $0.408 * *$ & 0.208 & $\underline{-0.478 *}$ & -0.152 & $0.4 * *$ \\
\hline $\begin{array}{c}(\text { Size } \\
\text { Difference })^{2}\end{array}$ & 0.000 & 0.004 & $\underline{-0.006 *}$ & -0.003 & $\underline{0.008 *}$ & 0.000 & $\underline{-0.006 *}$ \\
\hline $\begin{array}{c}\text { Color } \\
\text { Difference }\end{array}$ & $\underline{-0.014} *$ & $\underline{-0.019 * *}$ & -0.002 & 0.001 & -0.006 & 0.000 & 0.002 \\
\hline $\begin{array}{c}\text { Texture } \\
\text { Difference }\end{array}$ & 1.910 & -12.466 & 6.862 & -6.821 & 8.962 & 8.895 & 2.472 \\
\hline Text & $-4.198 * * *$ & $-6.605^{*} * *$ & $-2.732 * * *$ & -1.980 & -0.732 & 0.547 & -1.074 \\
\hline Human & $-2.166 *$ & $-5.598 * * *$ & 0.435 & $-7.946 * * *$ & $12.155 * * *$ & $-2.009 * *$ & -0.902 \\
\hline Animal & $-7.375 * * *$ & -4.346 & 14.51*** & -3.791 & $-5.787 * *$ & 0.51 & 1.909 \\
\hline
\end{tabular}

Table 6. Effect of text emotion and image emotion on number of backers (model 1)

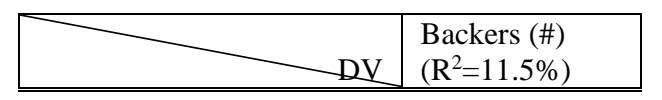

\begin{tabular}{|l|l|c|}
\hline \multicolumn{2}{|l|}{ IV } & \multicolumn{2}{|c|}{} \\
\hline \hline \multirow{3}{*}{$\begin{array}{l}\text { Picture } \\
\text { Emotion }\end{array}$} & Amusement & -1.861 \\
\cline { 2 - 3 } & Awe & -0.924 \\
\cline { 2 - 3 } & Contentment & $\underline{\mathbf{5 . 5 5 0} * * *}$ \\
\hline
\end{tabular}




\begin{tabular}{|l|l|c|}
\hline \multirow{4}{*}{} & Disgust & -1.774 \\
\cline { 2 - 3 } & Excitement & 0.174 \\
\cline { 2 - 3 } & Fear & $\underline{\mathbf{- 4 . 3 4 7} * *}$ \\
\cline { 2 - 3 } & Sadness & $\underline{\mathbf{6 . 3 8 0} * * *}$ \\
\hline \multirow{2}{*}{\begin{tabular}{l} 
Text $\begin{array}{l}\text { Description } \\
\text { Emotion }\end{array}$ \\
\cline { 2 - 3 }
\end{tabular}} & Anxiety & -6.896 \\
\cline { 2 - 3 } $\begin{array}{l}\text { Preset Goal (Control } \\
\text { Variable) }\end{array}$ & Sadness & $\underline{\mathbf{4 1 . 7 1 6} * *}$ \\
\hline $\begin{array}{l}\text { Keyword (Control } \\
\text { Variable) }\end{array}$ & $\underline{\mathbf{0 . 0 0 2} * * *}$ \\
\hline \multicolumn{2}{|c|}{ Note: $* * * \mathrm{p}<0.01, * * \mathrm{p}<0.05, * \mathrm{p}<0.1$} \\
\hline
\end{tabular}

Table 7. Effect of text emotion and image emotion on amount of fundraising (model 2)

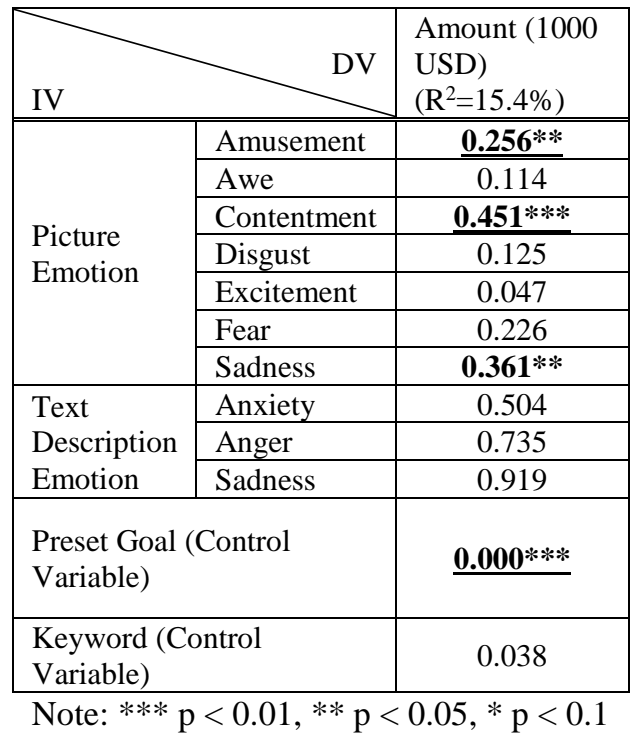

\subsection{Summary of results}

Table 4 and Table 5 lists all image attributes' effect on emotion while view title images. From the results, we find evidences that aesthetic attributes of image could truly predict emotions. Thus, H1 was supported partially.

We analyze the effect of both text and image emotion on the outcome of crowdfunding projects, and the results are in Table 6 and Table 7. We found that contentment is effective on all outcome metrics of crowdfunding projects, which includes number of backers and amount of fundraising (all $\mathrm{p}$ value < 0.01 , all coefficients are positive). Interestingly, sadness is effective from both image and text emotion on number of backer (biggest $\mathrm{p}$ value $<0.05$, all coefficients are positive). On top of that, sadness is also effective from image emotion on amount of fundraising and number of backers. The positive effect of sadness emotion on successfulness of fundraising is consistent with literature (Baberini et al. 2015; Small and Verrochi 2009). On the other hand, fear emotion feeling of images would lead to lower number of backers based on the result of our analysis. According to above results, $\mathrm{H} 2 \mathrm{a}$ and $\mathrm{H} 2 \mathrm{~b}$ are supported partially.

\subsection{Alternative Models: Direct and Mediation Models}

Some researches use images' attributes to directly predict the business outcome (e.g. clicks or CTR) in respective study setting (Goswami et al. 2011). Thus, we would like to compare the direct model (no emotions) and model with emotion. To compare two different models, we choose to use adj. $\mathrm{R}^{2}$ to compare. The results are shown in following able. From Table 9, we can tell that although there is subtle difference for model 2 , adj. $\mathrm{R}^{2}$ increase $2.5 \%$ for model 1 . There are 12 variables contribute to our dependent variable in proposed model and 18 variables contribute to our dependent variable in alternative model. Thus, our proposed model could better predict our dependent variable with a less sophisticated model.

Table 9 Model comparison

\begin{tabular}{|l|l|l|}
\hline \multirow{2}{*}{ Models } & $\begin{array}{l}\text { Model without } \\
\text { emotions }\end{array}$ & $\begin{array}{l}\text { Proposed } \\
\text { model }\end{array}$ \\
\cline { 2 - 3 } & adj. $\mathrm{R}^{2}$ & adj. $\mathrm{R}^{2}$ \\
\hline $\begin{array}{l}\text { Model 1 (Number } \\
\text { of backers) }\end{array}$ & $7.7 \%$ & $10.3 \%$ \\
\hline $\begin{array}{l}\text { Model 2 (Amount } \\
\text { of fundraising) }\end{array}$ & $14.1 \%$ & $14.0 \%$ \\
\hline
\end{tabular}

On the other hand, we also test the mediation to see if emotions mediate the relationship between image attributes and performance in the model. To test mediation, we employ Sobel test to test the possible mediation effect in our model. First step for Sobel test is test the direct effect of image attributes to crowdfunding performance. We only see few significant direct effects of image attributes on crowdfunding performance. Among all possible mediations, we only see few weak partial effects in our model. Thus, since we didn't find strong mediation in our model, we did not interpret any mediation effect in this study.

\section{Discussion}

\subsection{Practical implications}

The results of this research provide practical and actionable suggestions and insights to crowdfunding 
seekers and platforms to improve the performance/outcome of charity type crowdfunding projects.

Research results indicates that emotions are strong predictor of financial donation. But how to manipulate emotion is still questionable. This study provides practical instruction to those who are interesting in boosting the performance of crowdfunding projects. Prior studies show that certain emotions affect fundraising. Several studies have indicated that sadness would be a possible and powerful emotion in fundraising. Our findings are consistent with prior studies that sadness emotion could prompt people to provide financial aid to those in need (Liang et al. 2016; Small and Verrochi 2009). Thus, effect of sadness on fundraising performance has been verified by prior researches and this research. According to our results, warm hue, saturation and contrast of brightness are the attributes which affect the sadness emotion. Among these aesthetic attributes, warm hue, brightness, and contrast of brightness affect sadness emotion negatively. Therefore, decreasing those aesthetic attributes in title images could increasing the viewers' sadness emotion response and then may benefit crowdfunding performance. Thus, practitioner could intendedly manipulate warm hue, brightness, and contrast of brightness in setting up title images in crowdfunding projects to trigger possible sadness emotion response. Moreover, we also employ quadratic term to capture the effect of saturation and size difference, and the results indicate that overwhelming high or overwhelming low saturation would increase the sadness emotion. High saturation and low saturation will respectively make the photo extremely colorful or only grayscale. Professional photographer indicates that strong colors are relatively rare in the world, and those colors with medium saturated could bring the feeling of quieter or even pleasure (Freeman 2007). According to our results, extreme saturation is the setting we want to bring viewers the sadness emotion. On the other hand, high and low size difference between main body and background bring low sadness emotion, and medium size difference bring high sadness emotion. According to professional photographer's work, there is no particular way to set up the proportion of main body in photograph (Freeman 2007). But apparently, out results indicate that medium proportion of main body in photograph would bring higher sadness emotion.

Sadness is expected to be effective to crowdfunding fundraising performance. In addition to the finding of Small et al. (2009), contentment also significantly affects the performance of crowdfunding performance. Literature indicates that consumers who donate more are more contented than other who donate less (Krishna 2011), but we still don't know the relationship between contentment emotion and donation. Literature also argues that contentment is an important driver of reciprocal altruism, which means one try to help others and expect others will do the same in the future (Trivers 1971). Thus, we argue that when backers feel contented, they would like to help the others in need to enforce the backers' expected future contentment level due to reciprocal altruism. According to our results, rule of thirds, brightness, and animal in the image would affect contentment emotion positively, but diagonal dominance and text in image would affect contentment emotion negatively. Again, quadratic term helps us to capture the effect of saturation and size difference on contentment emotion. Regarding saturation, opposite to sadness emotion, medium saturation would bring higher contentment emotion, and high or low saturation would bring possible backers lower contentment emotion. It might be related to those possible positive feelings which medium saturation could bring to viewers (Freeman 2007). On top of that, size difference would affect contentment like size difference affect sadness, and medium proportion of main body would bring higher contentment.

Although manipulating these aesthetic attributes would possibly trigger not only sadness but also fear. In contrast to sadness, we found fear and amusement would negatively affect fundraising performance. Therefore, practitioner should manipulate aesthetic attributes more carefully.

\subsection{Theoretical implications}

Emotion is considered as important response for viewers who form their aesthetic appreciation from art works (Barry 2006; Carroll 2003). However, due to subjective characteristic of artworks, people always have subjective viewpoints toward artworks and make characteristics of artworks are hard to be captured and measured by researchers. Thanks to the work from researchers, although measurements may not be comprehensive to capture every characteristics of artworks, researches have provided a totally objective method to measure aesthetic attributes of photograph (Wang et al. 2013; Zhang et al. 2017). These objective aesthetic standards could be adopted by researcher to deal with subjective attributes of photograph in the future.

Existing researches provide evidences that emotion could be effective in making people provide financial aid to those in need in charity donation 
scenario (Baberini et al. 2015; Liang et al. 2016; Small and Verrochi 2009). This study is the first study to introduce emotion as possible motivator of financial aids in crowdfunding scenario. Different from charity donation, there is still no prior researches introduce emotion in crowdfunding scenario. Our results indicate that, similar to charity donation scenario, emotions would still be the significant predictors in crowdfunding performance, especially the effect of sadness is still as strong as results of previous literature. On the other hand, contentment unexpectedly affects the performance of crowdfunding significantly, contentment might need more researchers to discover the potential of contentment in fundraising.

To the best of our knowledge, this article is also the first article to introduce deep learning technique to analyze the emotion in crowdfunding scenario. Traditionally, emotions in image are hard to classify by objective and uniform standard. Thanks to the advantage neural network technique. Using computer to classify emotion in image is now possible. Our study shows the implication and value of deep learning technique for emotion and fundraising related research.

To summarize, this research introduces emotions into crowdfunding scenario which is the first for crowdfunding related research before. And our results indicate that emotions are still strong in motivating possible bakers to provide financial aid to achieve better crowdfunding performance. To predict possible emotions, this study is also the first research which employ objective standards to do so. This study not only consolidate the effectiveness of emotion but also introduce new and objective standard to measure aesthetic features which could affect emotions.

\subsection{Limitation and Future Research}

There are few limitations in our research. First, emotion feeling detection are limited to algorithm and software design (LIWC and deep learning). Although we use state-of-art and objective techniques to extract emotions, but since emotion feeling is subjective for every individual we might not be able to detect emotions accurately. Future research should try to use state-of-art techniques while they are available to better and accurately detect emotions.

On the other hand, we only focus on public benefit as our scope of research. The reason we focus on public benefit is due to its similarity to charity. The model and variables we propose and introduce in this research are based on existing literature of charity fundraising. Thus, public benefit is selected to be our research scope. Since we collected all of the crowdfunding projects under the public benefit category from Kickstarter.com, for robustness check, we tested the model with random selected subsamples from the data set. Future research should embark to collect data from a different crowdfunding platform.

\section{Conclusion}

For the researches of crowdfunding, we suggest that emotion might be critical based on existing charity researches. The results of this study also indicate the importance of emotion for crowdfunding research. This study provides example of using comprehensive tools and actionable and practical guides to crowdfunding seekers and platforms. We believe this study would be the pioneer to show IS scholars that how to implement new tools in researches and role of emotion in crowdfunding related researches. Finally, we expect more future researches would apply new tools in exploring their research area.

\section{References}

[1]Baberini, M., Coleman, C.-L., Slovic, P., and Västfjäll, D. 2015. "Examining the Effects of Photographic Attributes on Sympathy, Emotions, and Donation Behavior," Visual Communication Quarterly (22:2), pp. 118-128.

[2]Barry, A. M. 2006. "Perceptual Aesthetics: Transcendent Emotion, Neurological Image," Visual Communication Quarterly (13:3), pp. 134-151.

[3]Bland, E., S Black, G., and Lawrimore, K. 2007. "RiskReducing and Risk-Enhancing Factors Impacting Online Auction Outcomes: Empirical Evidence from EBay Auctions," Journal of Electronic Commerce Research (8).

[4]Burtch, G., Ghose, A., and Wattal, S. 2013. "An Empirical Examination of the Antecedents and Consequences of Contribution Patterns in Crowd-Funded Markets," Information Systems Research (24:3), pp. 499519 .

[5]Burtch, G., Ghose, A., and Wattal, S. 2016. "Secret Admirers: An Empirical Examination of Information Hiding and Contribution Dynamics in Online Crowdfunding," Information Systems Research (27:3), pp. 478-496.

[6]Carroll, N. 2003. "Art and Mood: Preliminary Notes and Conjectures,” The Monist (86:4), pp. 521-555.

[7]CHOI, J. N., SUNG, S. Y., LEE, K., and CHO, D.-S. 2011. "Balancing Cognition and Emotion: Innovation Implementation as a Function of Cognitive Appraisal and Emotional Reactions toward Innovation," Journal of Organizational Behavior (32:1), pp. 107-124. 
[8]Di, W., Sundaresan, N., Piramuthu, R., and Bhardwaj, A. 2014. Is a Picture Really Worth a Thousand Words? On the Role of Images in E-Commerce, presented at the International Conference on Web Search and Data Mining.

[9]Freeman, M. 2007. The Photographer's Eye: Composition and Design for Better Digital Photos, (1st edition.), Focal Press.

[10]Gilkeson, J. H., and Reynolds, K. 2003. "Determinants of Internet Auction Success and Closing Price: An Exploratory Study," Psychology and Marketing (20:6), pp. 537-566.

[11]Goswami, A., Chittar, N., and Sung, C. H. 2011. A Study on the Impact of Product Images on User Clicks for Online Shopping, presented at the International World Wide Web Conference 2011, Hyderabad, India.

[12]Jake Hobbs, Georgiana Grigore, and Mike Molesworth. 2016. "Success in the Management of Crowdfunding Projects in the Creative Industries," Internet Research (26:1), pp. 146-166.

[13]Kelly, K., Slater, M., and Karan, D. 2002. "Image Advertisements' Influence on Adolescents' Perceptions of the Desirability of Beer and Cigarettes," Journal of Public Policy \& Marketing (21:2), pp. 295-304.

[14]Krishna, A. 2011. "Can Supporting a Cause Decrease Donations and Happiness? The Cause Marketing Paradox," Journal of Consumer Psychology (21:3), pp. 338-345.

[15]Liang, J., Chen, Z., and Lei, J. 2016. "Inspire Me to Donate: The Use of Strength Emotion in Donation Appeals," Journal of Consumer Psychology (26:2), pp. 283-288.

[16]Lin, M., and Viswanathan, S. 2015. "Home Bias in Online Investments: An Empirical Study of an Online Crowdfunding Market," Management Science (62:5), pp. 1393-1414.

[17]Machajdik, J., and Hanbury, A. 2010. “Affective Image Classification Using Features Inspired by Psychology and Art Theory," in Proceedings of the 18th ACM International Conference on Multimedia, MM '10, New York, NY, USA: ACM, pp. 83-92.

[18]Mollick, E. 2014. "The Dynamics of Crowdfunding: An Exploratory Study," Journal of Business Venturing (29:1), pp. 1-16.

[19]Mollick, E., and Robb, A. 2016. "Democratizing Innovation and Capital Access: The Role of Crowdfinding," California Management Review (58:2), pp. 72-87.

[20]Ordanini, A., Miceli, L., Pizzetti, M., and A. Parasuraman. 2011. "Crowd-funding: Transforming Customers into Investors through Innovative Service Platforms," Journal of Service Management (22:4), pp. 443-470.Peck, J., and Childers, T. L. 2003. "To Have and to Hold: The Influence of Haptic Information on Product Judgments," Journal of Marketing (67:2), pp. 35-48.
[21]Pennebaker, J., Boyd, R., Jordan, K., and Blackburn, K. 2015. "The Development and Psychometric Properties of LIWC2015," Austin, TX: University of Texas at Austin.

[22]Ryu, S., and Kim, Y.-G. 2016. "A Typology of Crowdfunding Sponsors: Birds of a Feather Flock Together?," Electronic Commerce Research and Applications (16), pp. 43-54.

[23]Silvia, P. J. 2005. "Emotional Responses to Art: From Collation and Arousal to Cognition and Emotion," Review of General Psychology (9:4), pp. 342-357.

[24]Small, D. A., and Verrochi, N. M. 2009. "The Face of Need: Facial Emotion Expression on Charity Advertisements," Journal of Marketing Research (46:6), pp. 777-787.

[25]Thürridl, C., and Kamleitner, B. 2016. "What Goes Around Comes Around? REWARDS AS STRATEGIC ASSETS IN CROWDFUNDING," California Management Review (58:2), pp. 88-110.

[26]Trivers, R. L. 1971. "The Evolution of Reciprocal Altruism," The Quarterly Review of Biology (46:1), pp. 3557.

[27]USA Giving. 2017. “Giving USA 2017: Total Charitable Donations Rise to New High of \$390.05 Billion | Giving USA."

[28]Wang, X., Jia, J., Yin, J., and Cai, L. 2013. "Interpretable Aesthetic Features for Affective Image Classification," in 2013 IEEE International Conference on Image Processing, , September, pp. 3230-3234.

[29]Wood, S. L., and Moreau, C. P. 2006. "From Fear to Loathing? How Emotion Influences the Evaluation and Early Use of Innovations," Journal of Marketing (70:3), pp. 44-57.

[30]Yadollahi, A., Shahraki, A. G., and Zaiane, O. R. 2017. "Current State of Text Sentiment Analysis from Opinion to Emotion Mining," ACM Computing Surveys (50:2), 25:125:33.

[31]Yin, D., Bond, S. D., and Zhang, H. 2014. "Anxious or Angry? Effects of Discrete Emotions on the Perceived Helpfulness of Online Reviews," MIS Quarterly (38:2), pp. 539-560.

[32]Yuan, H., Lau, R. Y. K., and Xu, W. 2016. "The Determinants of Crowdfunding Success: A Semantic Text Analytics Approach," Decision Support Systems (91), pp. 67-76.

[33]Zhang, S., Lee, D., Singh, P. V., and Srinivasan, K. 2017. "How Much Is an Image Worth? Airbnb Property Demand Estimation Leveraging Large Scale Image Analytics," SSRN Scholarly Paper No. ID 2976021, SSRN Scholarly Paper, Rochester, NY: Social Science Research Network, May 25. 